Running head: INFLUENCE OF WORKING MEMORY

\title{
The Influence of Working Memory on the Anger Superiority Effect
}

Jun Moriya ${ }^{1}$, Ernst H. W. Koster ${ }^{2}$, \& Rudi De Raedt ${ }^{2}$

1: Rikkyo University, Japan

2: Ghent University, Belgium

Reference: Moriya, J., Koster, E.H.W., \& De Raedt, R. (in press). The influence of working memory on the anger superiority effect. Cognition \& Emotion.

Please note that this is the final word final. The final published paper may still contain difference so we kindly refer to the final print paper

Corresponding Author:

Jun Moriya

Mailing Address: 1-2-26, Kitano, Niiza-shi, Saitama, Japan 
Running head: INFLUENCE OF WORKING MEMORY

E-mail: jmoriya@rikkyo.ac.jp

Phone: $+81(0) 48-471-7046$

Fax: $+81(0) 48-471-7164$

\begin{abstract}
The anger superiority effect shows that an angry face is detected more efficiently than a happy face. However, it is still controversial whether attentional allocation to angry faces is a bottom-up process or not. We investigated whether the anger superiority effect is influenced by top-down control, especially working memory (WM). Participants remembered a color then searched for differently colored facial expressions. Just holding the color information in WM did not modulate the anger superiority effect. However, when increasing the probabilities of trials in which the color of a target face matched the color held in WM, participants were inclined to direct attention to the target face regardless of the facial expression. Moreover, the knowledge of high probability of valid trials eliminated the anger superiority effect. These results suggest that the anger superiority effect is modulated by top-down effects of WM, the probability of events and expectancy about these probabilities.
\end{abstract}

Keywords: working memory, visual search, facial expressions, emotion, attention 
Running head: INFLUENCE OF WORKING MEMORY

\section{The Influence of Working Memory on the Anger Superiority Effect}

Anger is one of the inherent human emotions. Anger expression is already observed in infancy (Braungart-Rieker, Hill-Soderlund, \& Karrass, 2010; Pemberton Roben et al., 2012), and the structure of anger is universal across cultures (Alonso-Arbiol et al., 2011). Because people express anger under interpersonal provocations or conflicts, rapid recognition of angry faces is important to restore relationships with others or to be motivated to avoid dangerous situations. Many previous studies have shown automatic attentional allocation to angry faces (Eastwood, Smilek, \& Merikle, 2001; Fox et al., 2000; Frischen, Eastwood, \& Smilek, 2008; Hansen \& Hansen, 1988; Horstmann \& Bauland, 2006; Öhman, Lundqvist, \& Esteves, 2001; Pinkham, Griffin, Baron, Sasson, \& Gur, 2010; Williams, Moss, Bradshaw, \& Mattingley, 2005). In these studies visual search paradigms are used in which several facial expressions are presented and participants are required to determine whether all presented facial expressions are from the same category (e.g., angry) or not. Reaction times (RTs) to detect an angry target, which is surrounded by happy faces, are shorter than those for a happy target in a crowd of angry distractor faces (Horstmann \& Bauland, 2006; Pinkham et al., 2010). This rapid detection of an angry face is called the anger superiority effect. Although some previous studies suggest that the anger superiority effect does not depend on the number of distractors (Öhman et al., 2001), it is still controversial whether attentional allocation to angry faces is a bottom-up and automatic attentional process or not (Frischen et al., 2008).

In recent studies, top-down effects on emotional processing have been demonstrated, suggesting that the anger superiority effect is not a purely bottom-up and automatic process. (Mohanty \& Sussman, 2013; Todd, Cunningham, Anderson, \& Thompson, 2012). Previous studies have shown that angry faces attract attention but that such attentional allocation is diminished when participants set their goals for detecting non-angry faces (Hahn \& Gronlund, 2007; Vogt, De Houwer, Crombez, \& Van Damme, 2013). Hahn and Gronlund (2007) 
Running head: INFLUENCE OF WORKING MEMORY

manipulated top-down goals by asking participants to search for a specific facial expression (i.e., angry or happy face). That is, participants voluntarily set a goal to find a specific facial expression. Although they found a more efficient search for angry-target faces as compared to happy-target faces, an angry face did not automatically capture attention when the top-down goal was to search for a happy face. These results indicate that the attentional priority for angry faces interacts with top-down and bottom-up influences. Moreover, they show an important role for top-down mechanisms in search tasks for facial expressions. These studies have shown that goal settings modify the attentional priority of angry faces. However, goal settings are not only the top-down factor that influences attentional priorities.

In recent years there has been increased interest in how working memory (WM) guides visual attention. Although recent studies have shown that the information maintained in WM guides visual attention to a non-emotional stimulus that matched the contents of WM (Olivers, Peters, Houtkamp, \& Roelfsema, 2011; Soto, Hodsoll, Rotshtein, \& Humphreys, 2008), only few studies investigated the effects of WM on visual search for emotional facial expressions (Grecucci, Soto, Rumiati, Humphreys, \& Rotshtein, 2010). In Soto, Heinke, Humphreys, and Blanco (2005), participants were required to remember a colored object (e.g., a red circle, a blue square) and then search for a tilted line among vertical lines. All lines were included in colored objects. In some trials, a colored object that matched the content of WM contained a tilted line inside of it (i.e., valid trials) while in other trials, a colored object that matched the content of WM contained a vertical line inside of it (i.e., invalid trials). Soto and colleagues showed that RTs in valid trials were shorter than those in invalid trials. This result suggests that participants efficiently direct attention to the stimuli matching the content of WM. That is, top-down effects of WM change attentional priority and suppress bottom-up effects in a visual search task. Based on these previous studies, it is possible that even for emotional facial expressions WM might have an effect on attentional allocation in a visual 
Running head: INFLUENCE OF WORKING MEMORY

search task and suppress the anger superiority effect in a top-down manner.

Grecucci et al. (2010) investigated the effects of WM on visual attention to facial expressions. Participants were asked to remember an emotional word (e.g., fearful, happy) and then search for a face of a particular gender, which showed fearful, happy, or neutral expressions. They could not find any effects of WM content on RTs. However, when the emotional word held in WM matched the target facial expression, the response in the visual cortex was much larger than that to a target face that did not match the content of WM. This result suggests that WM content influences visual perception of facial expressions. However, in this study, participants searched for a specific gender while the anger superiority effect is typically observed when people search for facial expressions (odd-one-out). They did not show angry faces and only two faces were presented in the search task. Therefore, it is still unclear whether WM content influences the anger superiority effect.

In the present study, we investigated whether the anger superiority effect was suppressed by manipulation of the content of WM. The present study focused on the interaction between bottom-up and top-down effects on the anger superiority effect. Although angry faces attract attention in a bottom-up manner (Hansen \& Hansen, 1988; Horstmann \& Bauland, 2006; Öhman et al., 2001), we cannot dismiss the top-down influence on visual search for facial expressions. Although there is convincing evidence for WM guidance of attention (Olivers et al., 2011; Soto et al., 2008), examining WM influences in the context of strong bottom-up capture of emotional information (leading to anger superiority effects) could reveal important information about the nature and conditions that are associated with WM guidance of attention.

The present experiment was modeled after previous studies that examined WM guidance of attention (Olivers, Meijer, \& Theeuwes, 2006; Soto et al., 2005). Participants were asked to remember a color of a circle for a later recognition test. Then, in a visual search 
Running head: INFLUENCE OF WORKING MEMORY

task, differently colored angry or happy faces (e.g., red angry face, blue happy face) were

presented and participants were required to determine whether all presented facial expressions were from the same category (e.g., angry) or one of them was from the different category (e.g., one angry face and five happy faces). The crucial point is that one different facial expression from the other five distractor faces matches the content of WM in some trials (i.e., valid trials) and does not in other trials (i.e., invalid trials). We hypothesized that in valid trials, participants might direct attention to the different facial expression regardless of angry or happy faces. However, if the anger superiority effect is sensitive to top-down influences, it should be reduced on valid trials.

\section{Experiment 1}

\section{Method}

\section{Participants}

Participants were 30 undergraduates aged between 18 and 38 years (mean age $=21.7$, $S D=3.8)$. They provided informed consent. All had normal or corrected-to-normal vision.

\section{Stimuli}

As working-memory stimuli, we used a colored circle of $0.9^{\circ}$ radius. It could be a red, blue, yellow, green, pink, or cyan circle. For the visual search task we used facial expressions taken from the standardized NimStim set (Tottenham et al., 2009). Sixteen identities (8 men and 8 women) displaying close-mouthed angry and happy expressions were used. Faces with closed mouths were used to prevent that perceptual differences would confound the effects. The faces also have a color (red, blue, yellow, green, pink, or cyan). The faces in the visual search task were adjusted to fit within an oval window. Mean luminance and contrast were matched for all faces. The facial size is $3.0^{\circ} \times 4.0^{\circ}$ in the visual search task.

The stimuli were presented on a 21 -inch monitor. The experiment was programmed using E-prime (Psychology Software Tools, Pittsburgh, PA). The viewing distance was about 
Running head: INFLUENCE OF WORKING MEMORY

$60 \mathrm{~cm}$. All stimuli were presented on a black background.

\section{Procedure}

A schematic depiction of an experiment trial is presented in Figure 1. A fixation cross appeared at the center of the black screen for $500 \mathrm{~ms}$, which was followed by a memory stimulus for $500 \mathrm{~ms}$. For the memory stimulus, a colored circle was presented at the center of the screen. Participants were required to remember the color of the circle for a later memory test, which was performed at the end of each trial. The color was randomly selected and each color was presented equally often.

After a 500-ms blank interval with a fixation cross, a visual search display was presented until participants responded. Six faces with different colors were positioned in a circle around fixation with a radius of $5.0^{\circ}$. They were all different identities. Half of them were male faces and the others were female faces. In target-present trials, one target face showed a different facial expression from the other five distractor faces (e.g., one happy face and five angry faces). In target-absent trials, all faces showed the same facial expression (e.g., all happy faces). Participants were asked to detect whether all the faces showed the same expression or one face showed an expression different from the others as accurately and as quickly as possible. Half of all trials were target-present trials, and the other half trials were target-absent trials. Moreover, in target-present trials, there were two trial types distinguished by the relationship between the contents of WM and color of the target. In half of targetpresent trials, the color of a target face matched the color held in WM (i.e., valid trials). In other half trials, the color of a non-target face matched the color held in WM (i.e., invalid trials). Taken together, probabilities of valid, invalid, and target-absent trials are $25 \%, 25 \%$, and 50\%, respectively. Happy and angry target as well distractor faces were randomly selected, presented equally often, in randomized order. The color of the faces was also randomly selected and each color of the target face was presented equally often. 
Running head: INFLUENCE OF WORKING MEMORY

After a response and 500-ms blank interval with a fixation cross, a colored circle was presented at the center of the screen in a memory recognition test. Participants were required to indicate whether the color of the circle was same or different from the memory cue, which they remembered at the first of each trial. Participants were instructed to respond by pressing the appropriate key on the computer keyboard as accurately as possible.

First, participants had 20 practice trials. If accuracy rates in practice trials were less than $70 \%$, participants were presented 20 additional practice trials until the accuracy rates was higher than $70 \%$. After the practice trials, participants completed 192 experimental trials.

\section{Results}

Data Preparation. We analyzed the data for each participants and calculated accuracy rates in both visual search and WM tasks. Each task had 6 conditions; angry target in valid trials, happy target in valid trials, angry target in invalid trials, happy target in invalid trials, all angry faces in target-absent trials, and all happy faces in target-absent trials. We excluded 5 participants because their accuracy rates were less than $70 \%$ in, at least, one of the six conditions in a visual search task.

Reaction Time Data in the Visual Search Task. Incorrect responses were excluded. Based on visual inspection of Boxplots, RTs shorter than $200 \mathrm{~ms}$ or longer than $7000 \mathrm{~ms}$ were removed from the data $(0.4 \%)$. Thereafter, we also excluded trials where RTs deviated more than three standard deviations from the individual mean for each participant (1.3\%).

We analyzed the RTs in the visual search task separately for target-present and targetabsent trials. For target-present trials (Figure 2A), a 2 (Working Memory: valid vs. invalid) $\times$ 2 (Target Face: angry vs. happy) ANOVA was used to examine our hypotheses. Significant main effects were observed on Working Memory, $F(1,24)=8.31, p<.01, \eta_{p}^{2}=.26$, and Target Face, $F(1,24)=20.03, p<.001, \eta_{p}^{2}=.46$. RTs in valid trials $(2058 \mathrm{~ms})$ were shorter than those in invalid trials $(2132 \mathrm{~ms})$. In addition, RTs for an angry target (2051 ms) were 
Running head: INFLUENCE OF WORKING MEMORY

shorter than those for a happy target $(2140 \mathrm{~ms})$. The interaction was not significant, $F(1,24)$ $=1.51, p=.70, \eta_{p}^{2}=.01$. Thus both the anger superiority effects and WM effects were observed independently.

We also calculated the anger superiority effect in valid and invalid trials, respectively, by subtracting RTs for an angry target from RTs for a happy target. Then, we compared them to investigate whether the anger superiority effect was influenced by WM effects. As shown by the abovementioned insignificant interaction effect, the anger superiority effect in valid trials $(78 \mathrm{~ms})$ was not significantly different from the effect in invalid trials $(100 \mathrm{~ms}), t(24)=$ $0.39, p=.70, d=0.13$. The anger superiority effect was observed in both valid trials, $t(24)=$ $2.42, p<.05, d=0.48$, and invalid trials, $t(24)=2.59, p<.05, d=0.52$ (using one sample ttests).

For target-absent trials, we compared RTs for all happy faces with those for all angry faces (Figure 2B). There was a significant difference between them, $t(24)=8.46, p<.001, d$ $=0.66$. RTs for happy faces $(2261 \mathrm{~ms})$ were shorter than those for angry faces $(2560 \mathrm{~ms})$.

Accuracy Rates in the Visual Search Task. We also conducted the same ANOVA and $\mathrm{t}$-test for accuracy rates for target-present and target-absent trials in the visual search task (Table 1). For target-present trials, a two-way ANOVA showed no significant main effects or interaction. For target-absent trials, a paired t-test showed significant difference between accuracy rates for happy and angry faces, $t(24)=7.27, p<.001, d=1.63$. Accuracy rates for all happy faces were higher than those for angry faces.

Accuracy Rates in the Working Memory Task. Finally, we conducted the same analyses for accuracy rates for target-present and target-absent trials in the working memory task (Table 1). For target-present trials, a two-way ANOVA showed no significant main effects and interaction (all $F \mathrm{~s}<1.75, p \mathrm{~s}>.19$ ). For target-absent trials, a paired samples $t$-test showed no significant difference between accuracy rates for happy and angry faces, $t(24)=$ 
Running head: INFLUENCE OF WORKING MEMORY $1.17, p=.25, d=0.32$.

Speed-Accuracy Trade-Off. We also investigated a speed-accuracy trade-off between RTs in the visual search task and accuracy rates in the working memory task. We conducted an analysis of covariance (ANCOVA) on RTs in the visual search task with accuracy rates in the working memory task as covariate for target-present trials. The results were the same as those in the ANOVA. We only found significant main effects of Working Memory, $F(1,23)=$ $8.88, p<.01, \eta_{p}^{2}=.26$, and Target Face, $F(1,24)=20.55, p<.001, \eta_{p}^{2}=.49$. We did not find any evidence for speed-accuracy trade-off.

\section{Discussion}

In Experiment 1, we investigated the effects of WM on the anger superiority effect. Considering that previous studies have shown that attention is attracted to non-emotional stimuli matching the contents of WM (Olivers et al., 2006, 2011; Soto et al., 2005, 2008), we predicted that participants would direct attention to a different facial expression from the other five distractor faces when the different facial expression had the same color of WM (i.e., valid trials). That is, in valid trials, participants would direct attention to a target face regardless of the facial expressions and the anger superiority might not be observed. However, contrary to our hypothesis, the anger superiority effect was present in valid trials and the effects did not differ from those in invalid trials. These results seem in line with the notion of automatic attentional allocation to angry faces. However, considering that RTs in invalid trials were longer than those in valid trials, WM did influence attentional allocation to angry faces. These results suggest that WM effects and the anger superiority effects were additive and WM did not overcome the anger superiority effect.

In target-absent trials, RTs for angry faces were longer than those for happy faces. This suggests that angry faces not only attract attention automatically but also hold attention, which is consistent with previous studies (Belopolsky, Devue, \& Theeuwes, 2011; Horstmann 
Running head: INFLUENCE OF WORKING MEMORY

\& Bauland, 2006; Horstmann, Scharlau, \& Ansorge, 2006; Koster, Crombez, Verschuere, \&

De Houwer, 2004). When an emotional face was presented in the center of the screen and participants disengaged attention from the face, attentional disengagement was delayed for an angry face compared to a neutral or a happy face (Belopolsky et al., 2011). The delayed attentional disengagement from angry faces was also observed in a visual search task (Horstmann \& Bauland, 2006; Horstmann et al., 2006). These results suggested that inefficient search for angry faces in a target-absent trials derives from difficulty in disengaging from angry faces.

The present results suggest that the anger superiority effect is a bottom-up and automatic attentional process and top-down WM effects do not suppress the anger superiority effect. However, top-down influences might not be an all-or-nothing phenomenon. In two subsequent experiments we manipulated the top-down influences to examine the interaction between bottom-up and top-down influences on attention.

An increased probability of valid trials amplifies the benefits of matching targets and has been found to enhance WM guidance of attention (Carlisle \& Woodman, 2011; Kiyonaga, Egner, \& Soto, 2012; Kuo, Chao, \& Yeh, 2013). In Carlisle and Woodman (2011), the task was almost same as the study of Soto and colleagues (2005). First, participants were required to remember a color of a stimulus, then in a visual search task, they reported whether a square with a gap up or down (i.e., a target) was presented among the four objects. They calculated the validity effect by subtracting the RTs in valid trials, in which color of a target matched the contents of WM, from RTs in neutral trials, in which a stimulus matching the color of WM was not presented in a visual search task. They showed that the validity effect in a highprobability condition, in which $80 \%$ of non-neutral trials were valid trials, was doubled compared to those in a low-probability condition, in which $20 \%$ of non-neutral trials were valid trials. In Experiment 1, half of all trials were target-present trials, and valid trials were 
Running head: INFLUENCE OF WORKING MEMORY

half of the target-present trials. That is, $25 \%$ of all trials were valid trials. Considering the previous studies (Carlisle \& Woodman, 2011; Kiyonaga et al., 2012; Kuo et al., 2013), the probabilities of valid trials in Experiment 1 were low. Therefore, the anger superiority effect might not be suppressed by WM influences. In Experiment 2 and 3, we increased the probability of valid trials and investigated whether the anger superiority effect remained present (like in Experiment 1) or was suppressed through enhanced top-down control elicited by increased probability of valid trials. In Experiment 2 and 3, 60\% of all trials were valid trials, $20 \%$ of all trials were invalid trials, and others were target-absent trials. In Experiment 2, participants were not informed about the probability while in Experiment 3, they were informed about the probability before the experiment started.

\section{Experiment 2}

\section{Method}

\section{Participants}

Participants were 31 undergraduates aged between 17 and 30 years (mean age $=20.9$, $S D=2.4$ ). They provided informed consent. All had normal or corrected-to-normal vision.

\section{Procedure}

The procedure was identical to that of Experiment 1, with the exception of the following modification. In the visual search task, $80 \%$ of all trials were target-present trials. In addition, three-fourths of target-present trials were valid trials. Taken together, probabilities of valid, invalid, and target-absent trials are $60 \%, 20 \%$, and 20\%, respectively. First, participants had 20 practice trials. If accuracy rates in practice trials were less than $70 \%$, participants were presented 20 additional practice trials until the accuracy rates became more than $70 \%$. After the practice trials, the participants completed 240 experimental trials.

\section{Results}

Data Preparation. We analyzed the data for each participants and calculated accuracy 
Running head: INFLUENCE OF WORKING MEMORY

rates in both visual search and WM tasks as Experiment 1. We excluded 1 participant because accuracy rates were lower than $70 \%$ in one of the six conditions in the visual search task.

Reaction Time Data in the Visual Search Task. Incorrect responses were excluded. Based on visual inspection of Boxplots, RTs shorter than $200 \mathrm{~ms}$ or longer than $7000 \mathrm{~ms}$ were removed from the data $(0.9 \%)$. We also excluded trials where RTs deviated more than three standard deviations from the individual mean for each participant (1.1\%).

We analyzed the RTs in the visual search task separately for target-present and targetabsent trials. In target-present trials (Figure 3A), a 2 (Working Memory: valid vs. invalid) $\times 2$ (Target Face: angry vs. happy) ANOVA showed significant main effects of Working Memory, $F(1,29)=26.73, p<.001, \eta_{p}^{2}=.48$, and Target Face, $F(1,29)=19.99, p<.001, \eta_{p}^{2}=.41$.

RTs in valid trials (1878 ms) were shorter than those in invalid trials (2062 ms). In addition, RTs for an angry target (1906 ms) were shorter than those for a happy target (2033 ms). The interaction was also significant, $F(1,29)=7.19, p<.05, \eta_{p}^{2}=.20$. RTs in valid trials were shorter than those in invalid trials for both an angry target (valid: $1841 \mathrm{~ms}$; invalid: $1971 \mathrm{~ms}$ ), $F(1,29)=11.60, p<.01, \eta_{p}^{2}=.29$, and a happy target (valid: $1914 \mathrm{~ms}$; invalid: $\left.2152 \mathrm{~ms}\right), F$ $(1,29)=30.05, p<.001, \eta_{p}^{2}=.51$. RTs for an angry target were also shorter than those for a happy target in both valid trials, $F(1,29)=10.08, p<.01, \eta_{p}^{2}=.26$, and invalid trials, $F(1$, 29) $=17.32, p<.001, \eta_{p}^{2}=.37$.

We also compared the anger superiority effect in valid trials with the effect in invalid trials. As shown by the abovementioned significant interaction effect, the anger superiority effect in valid trials $(73 \mathrm{~ms}$ ) was significantly different from the effect in invalid trials (181 $\mathrm{ms}), t(29)=2.68, p<.05, d=0.56$. However, the anger superiority effect was still observed in both valid trials, $t(29)=3.18, p<.01, d=0.58$, and invalid trials, $t(29)=4.16, p<.001, d$ $=0.76$.

For target-absent trials, we compared RTs for all happy faces with those for all angry 
Running head: INFLUENCE OF WORKING MEMORY

faces (Figure 3B). There was a significant difference between them, $t(29)=6.63, p<.001, d$ $=0.51$, with RTs for happy faces being shorter than those for angry faces.

Accuracy Rates in the Visual Search Task. We also conducted the same ANOVA and $t$-test for accuracy rates for target-present and target-absent trials in the visual search task (Table 1). For target-present trials, a two-way ANOVA showed only a main effect on Working Memory, $F(1,29)=7.70, p<.05, \eta_{p}^{2}=.21$. Accuracy rates in valid trials $(96.8 \%)$ were higher than those for invalid trials (95.1\%). For target-absent trials, a paired samples $t$-test showed significant difference between accuracy rates for happy and angry faces, $t(29)=6.29$, $p<.001, d=1.18$. Accuracy rates for all happy faces were higher than those for angry faces.

Accuracy Rates in the Working Memory Task. Finally, we conducted the same analyses for accuracy rates for target-present and target-absent trials in the working memory task (Table 1). For target-present trials, a two-way ANOVA showed no significant main effects and interaction (all $F_{\mathrm{s}}<2.34, p \mathrm{~s}>.13$ ). For target-absent trials, a paired samples $t$-test showed no significant difference between accuracy rates for happy and angry faces, $t(29)=$ $0.40, p=.69, d=0.09$.

Speed-Accuracy Trade-Off. We also investigated speed-accuracy trade-off between RTs in the visual search task and accuracy rates in the working memory task. We conducted ANCOVA on RTs in the visual search task with accuracy rates in the working memory task as covariate for target-present trials. The results were the same as those in ANOVA. We found significant main effects on Working Memory, $F(1,28)=22.40, p<.001, \eta^{2}{ }_{p}=.43$, and Target Face, $F(1,28)=19.18, p<.001, \eta_{p}^{2}=.40$, and interaction, $F(1,28)=4.36, p<.05$, $\eta_{p}^{2}=.14$. The results of further analyses were also same as those in ANOVA. Thus, we did not find any evidence for speed-accuracy trade-off.

\section{Discussion}

In Experiment 2, we investigated whether the increased probability of valid trials 
Running head: INFLUENCE OF WORKING MEMORY

enhanced the top-down effect and suppressed the anger superiority effect. In valid trials, the anger superiority effect was still observed, yet this effect was decreased compared to that in invalid trials. Participants might be inclined to direct attention to a stimulus that matches the content of WM regardless of facial expressions. However, angry faces still captured attention more strongly compared to happy faces. Hence, the anger superiority effect was reduced but still observed. In target-absent trials, RTs for angry faces were longer than those for happy faces, again. This might be due to difficulty in attentional disengagement from angry faces.

When comparing the anger superiority effect in Experiment 1 and 2, interestingly the magnitude of the anger superiority effect in valid trials were more similar than those in invalid trials (Valid trials: $78 \mathrm{~ms}$ in Experiment 1 and $73 \mathrm{~ms}$ in Experiment 2, Invalid trials: $100 \mathrm{~ms}$ in Experiment 1 and $181 \mathrm{~ms}$ in Experiment 2). This is consistent with previous studies, which showed that WM effects on visual attention were observed in invalid trials compared to valid trials when increasing the probability of the validity (Carlisle \& Woodman, 2011; Kuo et al., 2013). For instance, Kuo et al. (2013) showed that RTs in valid trials did not differ in low- and high-probability conditions. The validity effect in the high-probability condition was driven by slower RTs in invalid trials. They suggested that in high-probability condition, participants had difficulty in reorienting attention from distractors matching the contents of WM to a target. In the present study, participants might have difficulty in reorienting to a happy target after attention to angry distractors in invalid trials because of difficulty in disengaging from angry faces (Belopolsky et al., 2011; Koster et al., 2004). Therefore, in Experiment 2, the modulation of top-down effects on the anger superiority effect was observed by increased RTs in invalid trials.

The WM effect on attentional allocation is also subject to strategic control (Carlisle \& Woodman, 2011). Carlisle and Woodman did not change the probability of valid trials but they informed participants of fake probabilities. Participants who believed in a high 
Running head: INFLUENCE OF WORKING MEMORY

probability of valid trials showed a large WM effect on attentional allocation to a matching stimulus compared to participants who believed in a low probability. This result suggests that knowledge about the contingency between WM content and visual search could modulate attentional guidance by WM, enhancing attentional allocation to a stimulus matching the content of WM. In Experiment 1 and 2, participants did not know the probability of valid trials in advance. Therefore, an angry face might still capture attention and the anger superiority effect was observed. In Experiment 3, we informed participants of the probability of valid trials before the experiment started. We predicted that this knowledge would guide attention more efficiently towards a stimulus matching the contents of WM regardless of facial expressions, suppressing the anger superiority effect.

\section{Experiment 3}

\section{Method}

\section{Participants}

Participants were 19 undergraduates aged between 19 and 38 years (mean age $=22.3$, $S D=4.1$. They provided informed consent. All had normal or corrected-to-normal vision.

\section{Stimuli \& Procedure}

Stimuli and procedure were identical to those in Experiment 2, with the exception of the following modification. Participants were given instructions at the beginning of the experiment about the probability of valid, invalid, and target-absent trials.

\section{Results}

Data Preparation. We analyzed the data for each participants and calculated accuracy rates in both visual search and WM tasks as in the previous experiments. We excluded 2 participants because their accuracy rates were less than $70 \%$ in one of the six conditions in a visual search task.

Reaction Time Data in the Visual Search Task. Incorrect responses were excluded. 
Running head: INFLUENCE OF WORKING MEMORY

Based on visual inspection of Boxplots, RTs shorter than $200 \mathrm{~ms}$ or longer than $7000 \mathrm{~ms}$ were removed from the data $(0.1 \%)$. We also excluded trials where RTs deviated more than three standard deviations from the individual mean for each participant $(0.8 \%)$.

We analyzed the RTs in the visual search task separately for target-present and targetabsent trials. In target-present trials (Figure 4A), a 2 (Working Memory: valid vs. invalid) $\times 2$ (Target Face: angry vs. happy) ANOVA showed significant main effects of Working Memory, $F(1,16)=85.53, p<.001, \eta_{p}^{2}=.84$, and Target Face, $F(1,16)=7.85, p<.05, \eta_{p}^{2}=.33$. RTs in valid trials (1395 ms) were shorter than those in invalid trials (1759 ms). In addition, RTs for an angry target $(1536 \mathrm{~ms})$ were shorter than those for a happy target (1618 ms). The interaction was also significant, $F(1,16)=9.29, p<.01, \eta_{p}^{2}=.37$. RTs in valid trials were shorter than those in invalid trials for both an angry target (valid: $1391 \mathrm{~ms}$; invalid: $1680 \mathrm{~ms}$ ), $F(1,16)=48.75, p<.001, \eta_{p}^{2}=.75$, and happy target (valid: $1398 \mathrm{~ms}$; invalid: $\left.1838 \mathrm{~ms}\right), F$ $(1,16)=73.74, p<.001, \eta_{p}^{2}=.82$. RTs for an angry target were also shorter than those for a happy target in invalid trials, $F(1,16)=10.42, p<.01, \eta_{p}^{2}=.39$, whereas RTs for an angry and happy target did not significantly differ in valid trials, $F(1,16)=0.08, p=.79, \eta_{p}^{2}=.01$.

We also compared the anger superiority effect in valid trials with invalid trials. As shown by the abovementioned significant interaction effect, the anger superiority effect in valid trials $(7 \mathrm{~ms})$ was significantly different from the effect in invalid trials $(158 \mathrm{~ms}), t(16)=$ $3.05, p<.01, d=0.95$. Moreover, the anger superiority effect in valid trials was not significant anymore, $t(16)=0.28, p=.79, d=0.07$, whereas the effect in invalid trials was still significant, $t(16)=3.23, p<.01, d=0.78$.

For target-absent trials, we compared RTs for all happy faces with those for all angry faces (Figure 3B). There was a significant difference between them, $t(16)=6.20, p<.001, d$ $=0.66$. RTs for happy faces were shorter than those for angry faces.

Accuracy Rates in the Visual Search Task. We also conducted the same ANOVA and 
Running head: INFLUENCE OF WORKING MEMORY

$\mathrm{t}$-test for accuracy rates for target-present and target-absent trials in the visual search task

(Table 1). For target-present trials, a two-way ANOVA showed that only main effect of

Working Memory was marginally significant, $F(1,16)=4.38, p=.053, \eta_{p}^{2}=.22$. Accuracy rates in valid trials $(97.2 \%)$ were higher than those in invalid trials $(94.4 \%)$. For target-absent trials, a paired samples $t$-test showed significant difference between accuracy rates for happy and angry faces, $t(16)=3.82, p<.01, d=1.26$. Accuracy rates for all happy faces were higher than those for angry faces.

Accuracy Rates in the Working Memory Task. Finally, we conducted the same analyses for accuracy rates for target-present and target-absent trials in the working memory task (Table 1). For target-present trials, a two-way ANOVA showed no significant main effects and interaction (all $F_{\mathrm{s}}<2.77, p \mathrm{~s}>.11$ ). For target-absent trials, a paired t-test showed no significant difference between accuracy rates for happy and angry faces, $t(16)=1.14, p$ $=.27, d=0.24$.

Speed-Accuracy Trade-Off. We also investigated speed-accuracy trade-off between RTs in the visual search task and accuracy rates in the working memory task. We conducted ANCOVA on RTs in the visual search task with accuracy rates in the working memory task as covariate for target-present trials. The results were same as those in ANOVA. We found significant main effects of Working Memory, $F(1,15)=81.40, p<.001, \eta^{2}{ }_{p}=.83$, and Target Face, $F(1,15)=8.48, p<.01, \eta_{p}^{2}=.32$, and their interaction, $F(1,15)=9.55, p<.01, \eta_{p}^{2}$ $=.39$. The results of further analyses were also the same as those in the ANOVA. We did not find any evidence for speed-accuracy trade-off.

\section{Discussion}

In Experiment 3, we investigated whether knowledge about the color of the memory task predicting the target enhanced attentional guidance by WM and suppressed the anger superiority effect. In valid trials, the anger superiority was not observed. This result suggests 
Running head: INFLUENCE OF WORKING MEMORY

that in valid trials, participants might direct attention to the stimulus matching the contents of WM regardless of facial expressions. In invalid trials, the anger superiority effect was still observed. Because attention was not allocated to the target facial expression in invalid trials and an angry target might attract attention compared to a happy target, RTs for an angry target were shorter than those for a happy target. In target-absent trials, RTs for angry faces were longer than those for happy faces, again.

According to previous studies (Carlisle \& Woodman, 2011), knowledge of the probability of valid trials increases strategic control for visual search. This strategic control modulates attentional allocation and enhances attentional priority to targets that match the content of WM. Under these circumstances WM effects on visual search fully eliminated the anger superiority effect. Although previous studies proposed that the anger superiority effect was due to a bottom-up attentional process (Hansen \& Hansen, 1988; Horstmann \& Bauland, 2006; Öhman et al., 2001), top-down effects of WM can eliminate the effect.

Contrary to Experiment 2, the magnitude of the anger superiority effect was clearly suppressed in valid trials. In Experiment 3, participants were informed of the probability of valid trials. In such cases, participants might endogenously allocate attention to a stimulus matching the contents of WM. The endogenous top-down control enhances the detection of a target and RTs in valid trials in high-probability condition compared to those in lowprobability condition (Carlisle \& Woodman, 2011; Kiyonaga et al., 2012). In Kiyonaga et al. (2012), when participants were instructed that $100 \%$ of all trials were valid trials in a block, RTs in valid trials were faster than those in other conditions, in which the probability of valid trials was 50\%. The instruction of the probability of valid trials in Experiment 3 might enhance endogenous top-down control and have an effect on efficient detection of a target in valid trials.

\section{General Discussion}


Running head: INFLUENCE OF WORKING MEMORY

Angry faces are important signals in social situations. People rapidly direct attention to an angry face in a visual search task. This rapid detection of an angry face is called the anger superiority effect. However, it is still unclear whether the anger superiority effect is a bottomup and automatic process or not. As recent studies have shown that emotional processing is not necessarily an automatic process but can be influenced by top-down effects (Mohanty \& Sussman, 2013; Todd et al., 2012), the anger superiority effect might be also modulated by top-down control. The present study investigated whether manipulation of WM could guide attention to the stimulus matching the contents of WM regardless of facial expressions, thus suppressing the anger superiority effect. The present study revealed important boundary conditions to WM guidance of attention and the presence of the anger superiority effect. Experiment 1 showed that even when a facial expression had a feature which matched the content of WM, merely holding information in WM did not influence and suppress the anger superiority effect. However, with increasing probabilities of valid trials, in which a target face had the same feature (i.e., color) as the contents of WM, the top-down effect decreased the anger superiority effect (Experiment 2). Moreover, the knowledge of high probability of valid trials eliminated the anger superiority effect (Experiment 3). These results suggest that an angry face strongly attracts attention in a bottom-up manner despite this still being susceptible to top-down control.

WM effects on the anger superiority effect were not observed under low probability of valid trials, and a high probability was necessary to manipulate attentional allocation to facial expressions. However, in the previous studies, which investigated the WM effect on attention to non-emotional stimuli, even under low probability conditions, participant directed attention to the stimulus matching the content of WM (Olivers et al., 2006, 2011; Soto et al., 2005, 2008). In these studies, a non-emotional target was used and in the absence of salient stimuli that capture attention, attention is strongly determined by top-down factors. Contrary to these 
Running head: INFLUENCE OF WORKING MEMORY

studies, an angry face is more salient and attracts attention (Eastwood et al., 2001; Fox et al., 2000; Frischen et al., 2008; Hansen \& Hansen, 1988; Horstmann \& Bauland, 2006; Öhman et al., 2001; Pinkham et al., 2010; Williams et al., 2005). The attentional priority of angry faces competes with WM guidance of attention. Under such circumstances, WM guidance of attention does not always reduce the anger superiority effect. Only a high probability of valid trials enhanced the effects of WM in our study and then WM guidance suppressed the anger superiority effect.

In the current study we found that the suppression of attentional allocation to emotional faces depends on the strength of top-down guidance of attention. This is in line with previous studies already showing that emotion processing is reduced under circumstances where attentional resources are limited (Pessoa, Padmala, \& Morland, 2005; Yates, Ashwin, \& Fox, 2010). When attentional demands are high and attentional resources are fully exhausted to some stimuli, attention is not allocated to emotional stimuli and emotion processing is not observed. When attentional demands are low, emotion processing is observed even for unattended emotional stimuli. Based on the present and previous studies it is clear that not all levels of top-down attentional control are sufficient to suppress emotional processing. Suppression of emotional information requires strong top-down control.

The current study highlights the dynamic interplay between bottom-up and top-down influences on visual search. The current findings are relevant to theories of WM guidance of visual search as results of the current study show that in complex environments with emotionally relevant information, WM guidance is less efficient and does not reduce attentional capture by emotional information. Only holding relevant non-emotional features of targets in WM did not suppress the bottom-up processing of facial expressions because angry faces strongly attract attention. Top-down effects of WM were observed when the probability of valid trials was high and/or knowledge of such probabilities prioritized attention to relevant 
Running head: INFLUENCE OF WORKING MEMORY

information. Because knowledge and expectations facilitate processing of upcoming stimuli related to the expectations (Mohanty \& Sussman, 2013), top-down effects would enhance the saliency of relevant stimuli and suppress the anger superiority effect. Although some studies have focused on bottom-up effects (e.g., perceptual saliency) on visual search for facial expressions (Horstmann \& Bauland, 2006), the present study elucidated the important role of top-down effects on the anger superiority effect.

Previous studies have shown that goal settings suppress the attentional priority of angry faces (Hahn \& Gronlund, 2007; Vogt et al., 2013) while the present study suggest that, under certain conditions, information in WM also suppresses the anger superiority effect. Olivers et al. (2011) proposed that even when a stimulus held in WM is not a target in a visual search task, it would receive the status of search template for the upcoming task. That is, the goal setting for a target on search template might be replaced with the stimulus held in WM. In the present study, it is possible that WM had an effect on goal settings. Especially in Experiment 3, participants were informed the probabilities of valid trials before the experiment started. This knowledge might enhance the effects of goal settings. The present results suggest that goal-settings might change indirectly by manipulating contents of WM.

The present results can be related to the role of WM in the biased competition model (Desimone \& Duncan, 1995). According to the biased competition model, several objects in the visual input compete with each other for neural representations. A few of highly activated neural representations could gain access to attentional processing. Desimone and Duncan (1995) proposed that WM increases neural activity of WM-relevant stimuli. This top-down bias increases the probability that those stimuli matching the content of WM could win among several neural presentations. Then, stimuli in WM might become a focus of attention. However, threatening stimuli, such as angry faces, also activate the neural representations in a bottom-up fashion (Lindquist, Wager, Kober, Bliss-Moreau, \& Barrett, 2012). Top-down WM 
Running head: INFLUENCE OF WORKING MEMORY

effects and bottom-up emotional effects might compete with each other in the present study, and the competition might have an effect on the anger superiority effect. When the activation of top-down WM effects is comparable with that of bottom-up emotional effects like in Experiment 1, neither of them wins and both effects are observed equally. If the activation of WM effects is bigger than that of emotional effects, top-down WM could overcome the anger superiority effect.

In previous studies on top-down effects on emotional processing, attentional manipulation was a focus of interest (Pessoa et al., 2005; Yates et al., 2010). They investigated whether emotional stimuli attracted attention when participants were required to other nonemotional stimuli or features. However, several factors modify the attentional priority, such as goal-settings and motivation (Hahn \& Gronlund, 2007; Pessoa, 2009; Vogt et al., 2013). Although WM is strongly related to attentional priority (Desimone \& Duncan, 1995) and visual WM can be defined as the active maintenance of attention to visual stimuli (Chun, 2011), the role of WM on emotional processing in visual attention has received scarce research. In previous studies on emotional processing, WM was manipulated as a load, which enhanced attentional control and increased the suppression of emotional processing (MacNamara, Schmidt, Zelinsky, \& Hajcak, 2012; Van Dillen \& Derks, 2012). In these studies, the contents of WM were not important. The present study suggests not only WM load but also WM contents play an important role for attentional allocation and emotional processing.

There is a limitation in the present study. Because there were no neutral trials, in which a stimulus matching the color of WM was not presented in a visual search task, it is difficult to conclude that the top-down effect was suppressing detecting an angry target in happy distractors or enhancing detection of a happy target in angry distractors. In future experiments, we need to set neutral trials. If the top-down effect is suppressing detection of an 
Running head: INFLUENCE OF WORKING MEMORY

angry target, RTs for an angry target in valid trials will be comparable with or longer than those in neutral trials. On the other hand, if the top-down effect is enhancing detection of a happy target in angry distractors, RTs for a happy target in valid trials will be shorter than those in neutral trials.

In sum, the present study has shown that the anger superiority effect can be suppressed by WM influences. Because angry faces strongly attract attention, just holding information, which is related to a target, in WM did not suppress the anger superiority effect. However, when increasing the probabilities that a target facial expression had a feature matching the contents of WM, the effect of WM was enhanced and the anger superiority effect was diminished. Moreover, knowledge of high probability of valid trials eliminated the anger superiority effect completely. The current study highlights the dynamic interplay between bottom-up and top-down influences on visual search. 


\section{References}

Alonso-Arbiol, I., Van De Vijver, F. J. R., Fernandez, I., Paez, D., Campos, M., \& Carrera, P. (2011). Implicit theories about interrelations of anger components in 25 countries. Emotion, 11(1), 1-11. doi:10.1037/a0020295

Belopolsky, A. V., Devue, C., \& Theeuwes, J. (2011). Angry faces hold the eyes. Visual Cognition, 19(1), 27-36. doi:10.1080/13506285.2010.536186

Braungart-Rieker, J. M., Hill-Soderlund, A. L., \& Karrass, J. (2010). Fear and anger reactivity trajectories from 4 to 16 months: the roles of temperament, regulation, and maternal sensitivity. Developmental Psychology, 46(4), 791-804. doi:10.1037/a0019673

Carlisle, N. B., \& Woodman, G. F. (2011). Automatic and strategic effects in the guidance of attention by working memory representations. Acta Psychologica, 137(2), 217-225. doi:10.1016/j.actpsy.2010.06.012

Chun, M. M. (2011). Visual working memory as visual attention sustained internally over time. Neuropsychologia, 49(6), 1407-1409. doi:10.1016/j.neuropsychologia.2011.01.029

Desimone, R., \& Duncan, J. (1995). Neural mechanisms of selective visual attention. (M. Cowan, Ed.)Annual Review of Neuroscience, 18(1), 193-222. doi:10.1146/annurev.ne.18.030195.001205

Eastwood, J. D., Smilek, D., \& Merikle, P. M. (2001). Differential attentional guidance by unattended faces expressing positive and negative emotion. Perception and Psychophysics, 63(6), 1004-1013. doi:10.3758/BF03194519

Fox, E., Lester, V., Russo, R., Bowles, R. J., Pichler, A., \& Dutton, K. (2000). Facial expressions of emotion: Are angry faces detected more efficiently? Cognition \& Emotion, 14(1), 61-92. doi:10.1080/026999300378996

Frischen, A., Eastwood, J. D., \& Smilek, D. (2008). Visual search for faces with emotional expressions. Psychological Bulletin, 134(5), 662-676. doi:10.1037/0033-2909.134.5.662

Grecucci, A., Soto, D., Rumiati, R. I., Humphreys, G. W., \& Rotshtein, P. (2010). The interrelations between verbal working memory and visual selection of emotional faces. Journal of Cognitive Neuroscience, 22(6), 1189-1200. doi:10.1162/jocn.2009.21276

Hahn, S., \& Gronlund, S. D. (2007). Top-down guidance in visual search for facial expressions. Psychonomic Bulletin \& Review, 14(1), 159-165. doi:10.3758/BF03194044

Hansen, C. H., \& Hansen, R. D. (1988). Finding the face in the crowd: An anger superiority effect. Journal of Personality and Social Psychology, 54(6), 917-924. doi:10.1037/00223514.54.6.917

Horstmann, G., \& Bauland, A. (2006). Search asymmetries with real faces: Testing the angersuperiority effect. Emotion, 6(2), 193-207. doi:10.1037/1528-3542.6.2.193 
Running head: INFLUENCE OF WORKING MEMORY

Horstmann, G., Scharlau, I., \& Ansorge, U. (2006). More efficient rejection of happy than of angry face distractors in visual search. Psychonomic Bulletin \& Review, 13(6), 10671073. doi:10.3758/BF03213927

Kiyonaga, A., Egner, T., \& Soto, D. (2012). Cognitive control over working memory biases of selection. Psychonomic Bulletin \& Review, 19(4), 639-646. doi:10.3758/s13423-0120253-7

Koster, E. H. W., Crombez, G., Verschuere, B., \& De Houwer, J. (2004). Selective attention to threat in the dot probe paradigm: Differentiating vigilance and difficulty to disengage. Behaviour Research and Therapy, 42(10), 1183-1192. doi:10.1016/j.brat.2003.08.001

Kuo, C.-Y., Chao, H.-F., \& Yeh, Y.-Y. (2013). Strategic control modulates working memorydriven attentional capture. Experimental Psychology, 60(1), 3-11. doi:10.1027/1618$3169 / \mathrm{a} 000167$

Lindquist, K. a, Wager, T. D., Kober, H., Bliss-Moreau, E., \& Barrett, L. F. (2012). The brain basis of emotion: A meta-analytic review. Behavioral and Brain Sciences, 35(3), 121143. doi:10.1017/S0140525X11000446

MacNamara, A., Schmidt, J., Zelinsky, G. J., \& Hajcak, G. (2012). Electrocortical and ocular indices of attention to fearful and neutral faces presented under high and low working memory load. Biological Psychology, 91(3), 349-356.

doi:10.1016/j.biopsycho.2012.08.005

Mohanty, A., \& Sussman, T. J. (2013). Top-down modulation of attention by emotion. Frontiers in Human Neuroscience, 7, 102. doi:10.3389/fnhum.2013.00102

Öhman, A., Lundqvist, D., \& Esteves, F. (2001). The face in the crowd revisited: A threat advantage with schematic stimuli. Journal of Personality and Social Psychology, 80(3), 381-396. doi:10.1037/0022-3514.80.3.381

Olivers, C. N. L., Meijer, F., \& Theeuwes, J. (2006). Feature-based memory-driven attentional capture: Visual working memory content affects visual attention. Journal of Experimental Psychology: Human Perception and Performance, 32(5), 1243-1265. doi:10.1037/0096-1523.32.5.1243

Olivers, C. N. L., Peters, J., Houtkamp, R., \& Roelfsema, P. R. (2011). Different states in visual working memory: When it guides attention and when it does not. Trends in Cognitive Sciences, 15(7), 327-334. doi:10.1016/j.tics.2011.05.004

Pemberton Roben, C. K., Bass, A. J., Moore, G. a., Murray-Kolb, L., Tan, P. Z., Gilmore, R. O., ... Teti, L. O. (2012). Let me go: The influences of crawling experience and temperament on the development of anger expression. Infancy, 17(5), 558-577. doi:10.1111/j.1532-7078.2011.00092.x

Pessoa, L. (2009). How do emotion and motivation direct executive control? Trends in Cognitive Sciences, 13(4), 160-166. doi:10.1016/j.tics.2009.01.006 
Running head: INFLUENCE OF WORKING MEMORY

Pessoa, L., Padmala, S., \& Morland, T. (2005). Fate of unattended fearful faces in the amygdala is determined by both attentional resources and cognitive modulation. NeuroImage, 28(1), 249-255. doi:10.1016/j.neuroimage.2005.05.048

Pinkham, A. E., Griffin, M., Baron, R., Sasson, N. J., \& Gur, R. C. (2010). The face in the crowd effect: anger superiority when using real faces and multiple identities. Emotion, 10(1), 141-146. doi:10.1037/a0017387

Soto, D., Heinke, D., Humphreys, G. W., \& Blanco, M. J. (2005). Early, involuntary topdown guidance of attention from working memory. Journal of Experimental Psychology: Human Perception and Performance, 31(2), 248-261. doi:10.1037/0096-1523.31.2.248

Soto, D., Hodsoll, J., Rotshtein, P., \& Humphreys, G. W. (2008). Automatic guidance of attention from working memory. Trends in Cognitive Sciences, 12(9), 342-348. doi:10.1016/j.tics.2008.05.007

Todd, R. M., Cunningham, W. A., Anderson, A. K., \& Thompson, E. (2012). Affect-biased attention as emotion regulation. Trends in Cognitive Sciences, 16(7), 365-372. doi:10.1016/j.tics.2012.06.003

Tottenham, N., Tanaka, J. W., Leon, A. C., McCarry, T., Nurse, M., Hare, T. A., ... Nelson, C. (2009). The NimStim set of facial expressions: Judgments from untrained research participants. Psychiatry Research, 168(3), 242-249. doi:10.1016/j.psychres.2008.05.006

Van Dillen, L. F., \& Derks, B. (2012). Working memory load reduces facilitated processing of threatening faces: An ERP study. Emotion, 12(6), 1340-1349. doi:10.1037/a0028624

Vogt, J., De Houwer, J., Crombez, G., \& Van Damme, S. (2013). Competing for attentional priority: Temporary goals versus threats. Emotion, 13(3), 587-598. doi:10.1037/a0027204

Williams, M., Moss, S., Bradshaw, J., \& Mattingley, J. (2005). Look at me, I'm smiling: Visual search for threatening and nonthreatening facial expressions. Visual Cognition, 12(1), 29-50. doi:10.1080/13506280444000193

Yates, A., Ashwin, C., \& Fox, E. (2010). Does emotion processing require attention? The effects of fear conditioning and perceptual load. Emotion, 10(6), 822-830. doi: $10.1037 / \mathrm{a} 0020325$ 
Running head: INFLUENCE OF WORKING MEMORY

Acknowledgments

We thank Lynn Bruyneel, Inez Greven, and Tobias Six for their help for experiments.

We also thank all PANLAB members in Ghent University for helpful comments on our experiments.

Preparation of this paper was supported by Grant BOF10/GOA/014 for a Concerted Research Action of Ghent University (awarded to Rudi De Raedt and Ernst Koster), and the Japan Society for the Promotion of Science (JSPS): Grant-in-Aid for JSPS Fellows (10J06078 to Jun Moriya). 
Running head: INFLUENCE OF WORKING MEMORY

Figure Captions

Figure 1. Example of the visual displays in an invalid trial in Experiment 1.

Figure 2. Results in the visual search task in Experiment 1. (A) Mean RTs in target-present trials. (B) Mean RTs in target-absent trials. Error bars represent within-subjects standard errors of the means (Cousineau, 2005).

Figure 3. Results in the visual search task in Experiment 2. (A) Mean RTs in target-present trials. (B) Mean RTs in target-absent trials.

Figure 4. Results in the visual search task in Experiment 3. (A) Mean RTs in target-present trials. (B) Mean RTs in target-absent trials. 


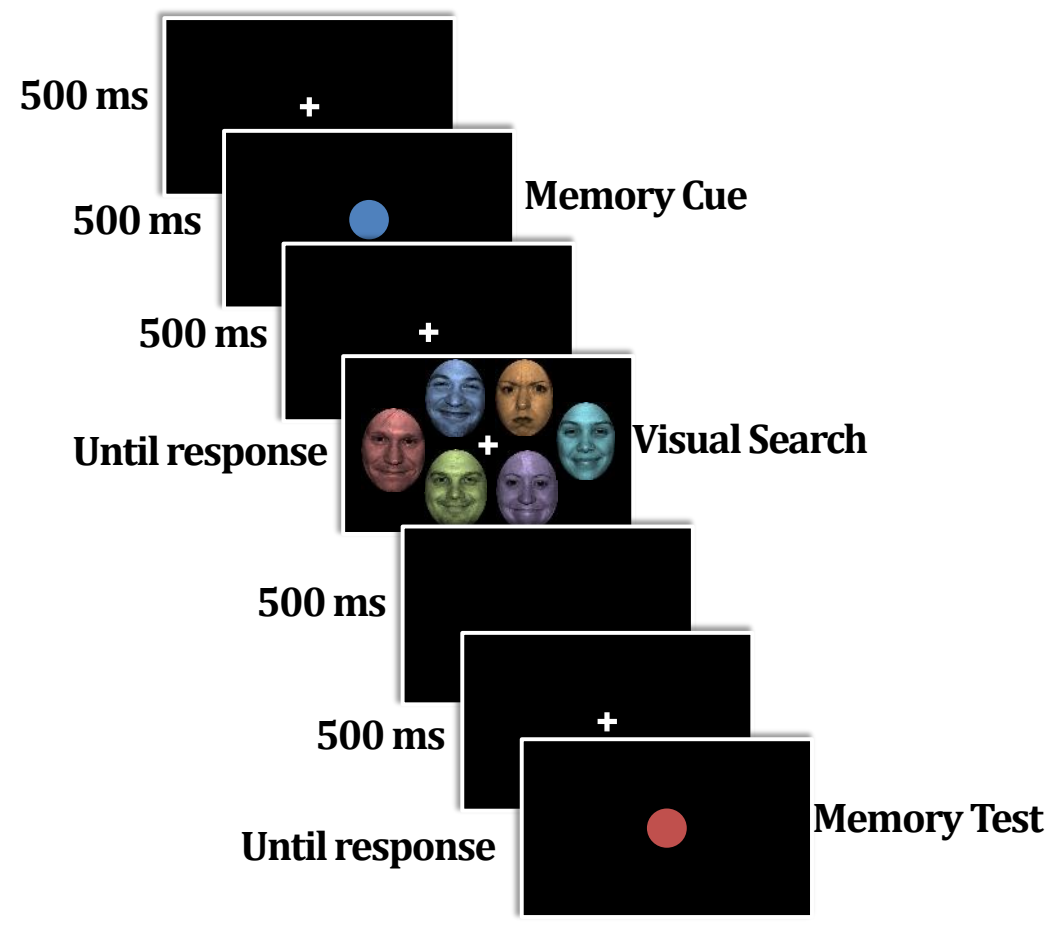


Running head: INFLUENCE OF WORKING MEMORY

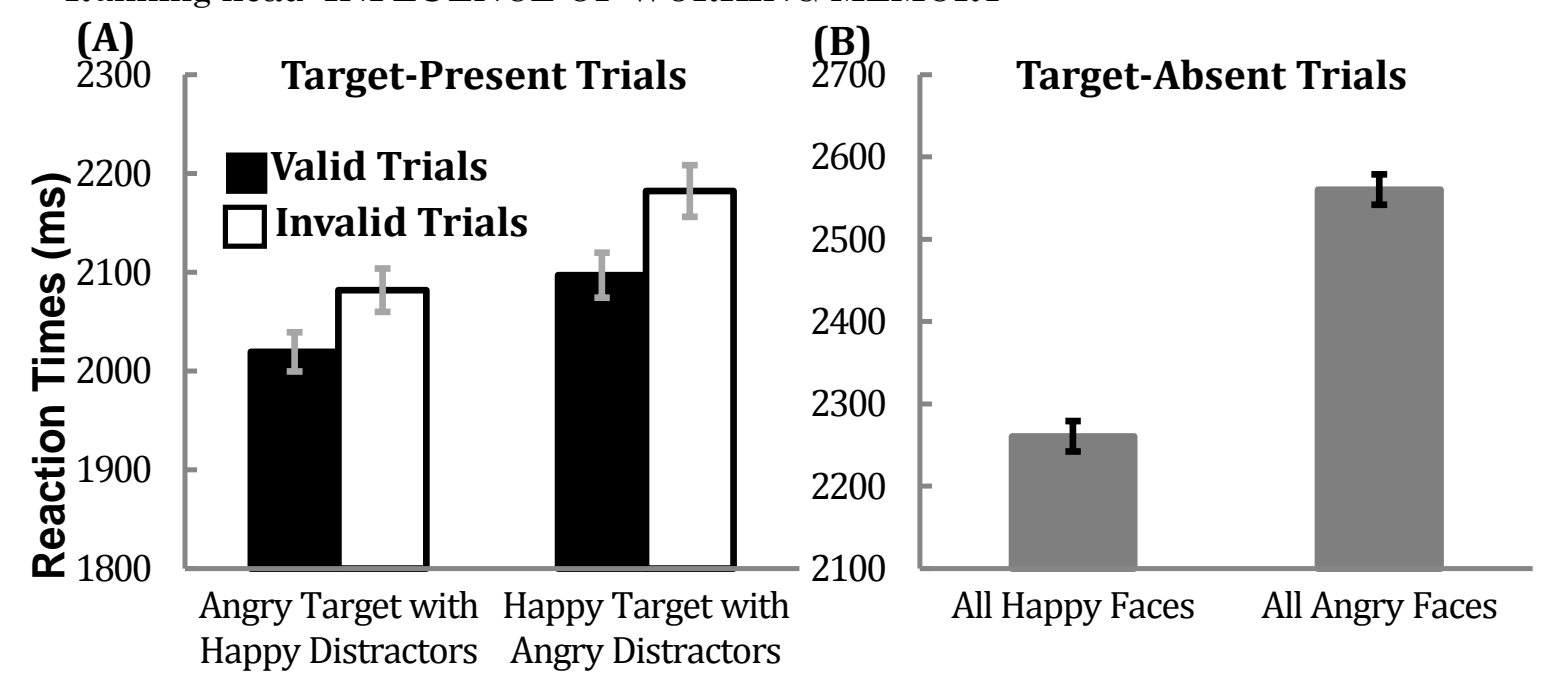


Running head: INFLUENCE OF WORKING MEMORY

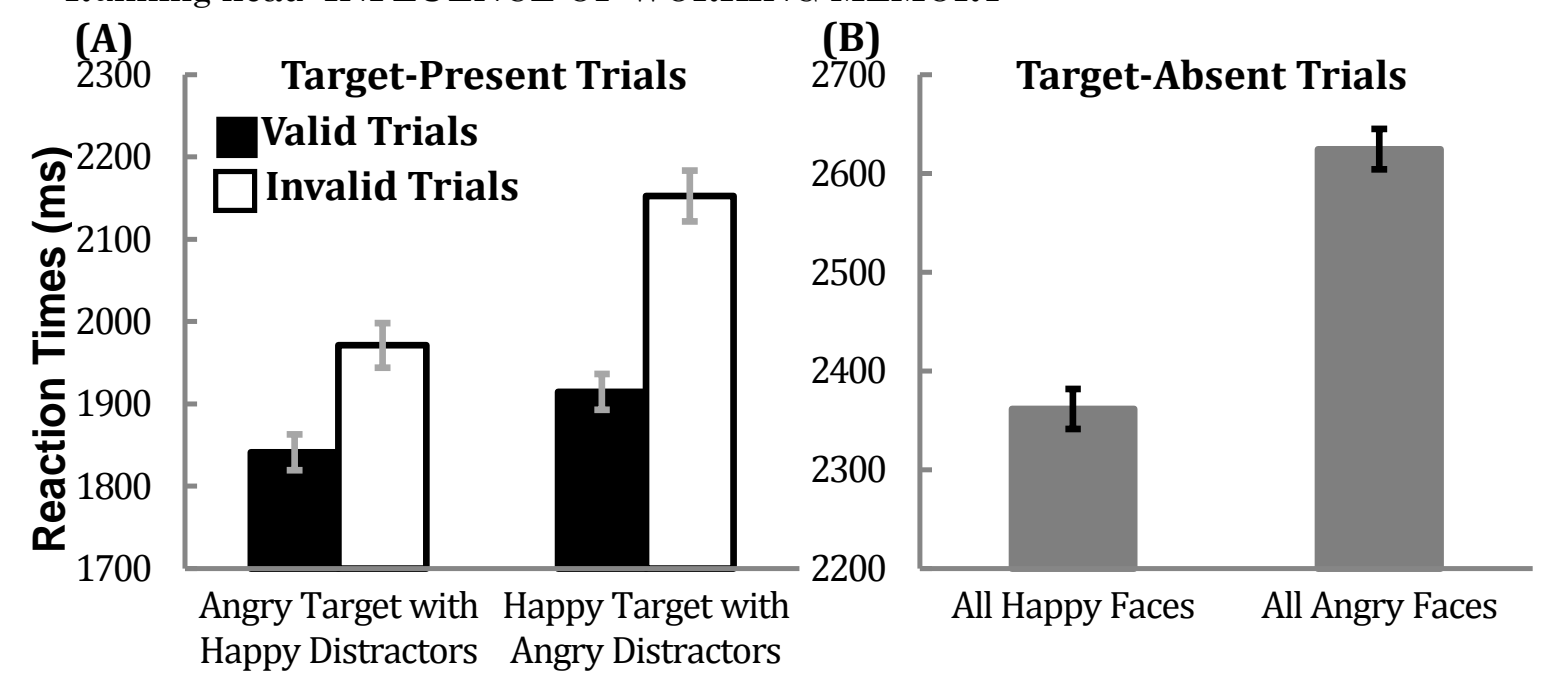


Running head: INFLUENCE OF WORKING MEMORY

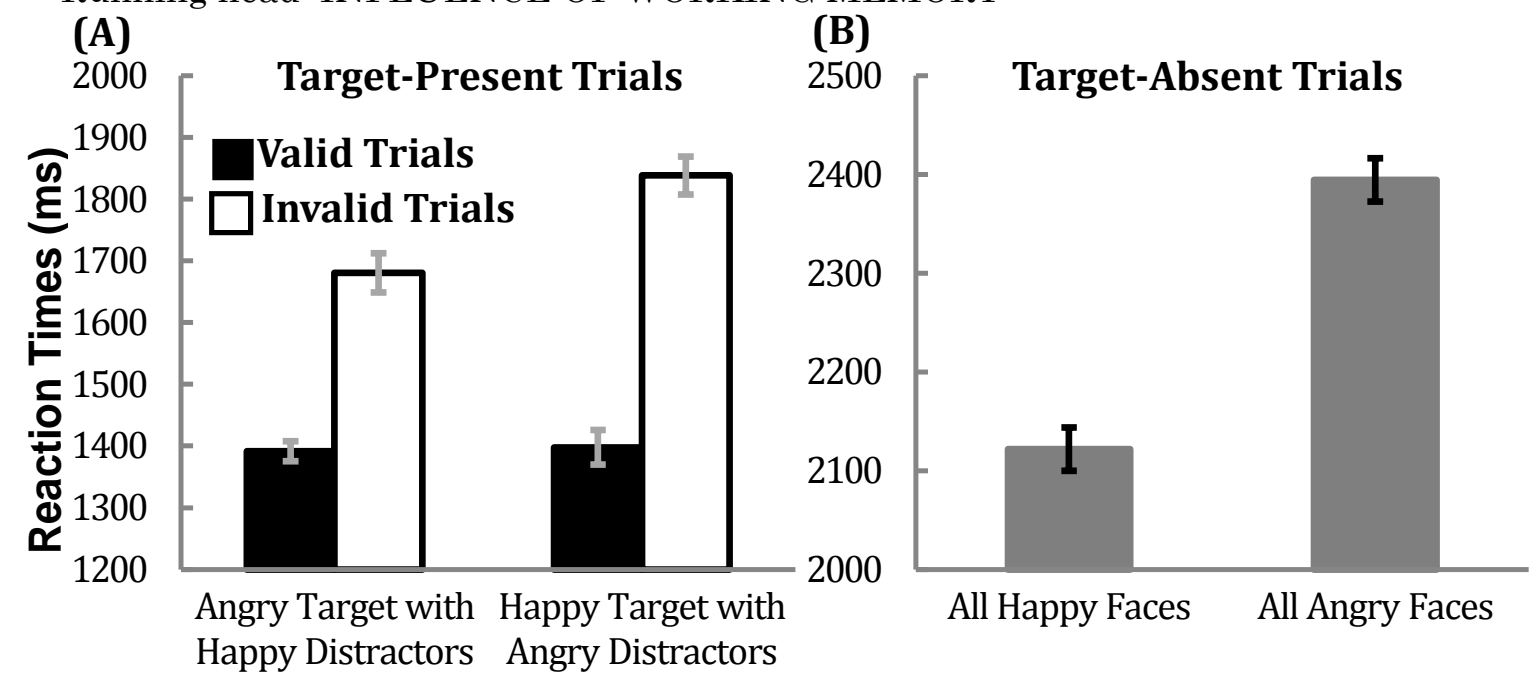

